\title{
EchoGéo
}

$8 \mid 2009$

Moyen-Orient : conflits et mobilités dans un espace mondialisé

\section{L'exil irakien à Damas}

Modes d'insertion urbaine et reconfiguration des réseaux migratoires

\section{Mohamed Kamel Doraï}

\section{(2) OpenEdition \\ Journals}

Édition électronique

URL : https://journals.openedition.org/echogeo/10976

DOI : 10.4000/echogeo.10976

ISSN : 1963-1197

Éditeur

Pôle de recherche pour l'organisation et la diffusion de l'information géographique (CNRS UMR 8586)

Référence électronique

Mohamed Kamel Doraï, « L'exil irakien à Damas », EchoGéo [En ligne], 8 | 2009, mis en ligne le 31 mars 2009, consulté le 31 juillet 2021. URL : http://journals.openedition.org/echogeo/10976 ; DOI : https:// doi.org/10.4000/echogeo.10976

Ce document a été généré automatiquement le 31 juillet 2021.

EchoGéo est mis à disposition selon les termes de la licence Creative Commons Attribution - Pas d'Utilisation Commerciale - Pas de Modification 4.0 International (CC BY-NC-ND) 


\title{
L'exil irakien à Damas
}

\author{
Modes d'insertion urbaine et reconfiguration des réseaux migratoires
}

\author{
Mohamed Kamel Doraï
}

\section{Introduction}

1 L'image véhiculée par les mouvements forcés de population dans les pays dits du Sud est souvent associée aux camps de réfugiés ainsi qu'à l'intervention humanitaire médiatisée lors des crises graves. La question des réfugiés urbains est, elle, beaucoup moins présente, moins visible, même si elle émerge de façon croissante dans le champ scientifique depuis quelques années. Selon le Haut Commissariat aux Réfugiés des Nations Unies (HCR) près d'un cinquième des réfugiés sont installés actuellement dans des espaces urbains ${ }^{1}$. Au Moyen-Orient, où se concentrent aujourd'hui plusieurs millions de réfugiés et déplacés (principalement Palestiniens, Soudanais et Irakiens), la population réfugiée est majoritairement urbaine que ce soit au Caire, à Amman, Beyrouth ou Damas. Dans le même temps, l'ensemble de la région connaît un développement urbain rapide, dont les migrants - internes et internationaux, forcés ou non - sont l'un des principaux moteurs (Al-Ali, 2004).

2 L'agglomération damascène n'échappe pas à la règle, et sa croissance repose en grande partie sur l'arrivée de nouvelles populations tant syrienne qu'étrangère, qui s'installent aux marges de la ville. Depuis 1948, pour ne pas remonter aux vagues antérieures à l'indépendance du pays, Damas est un espace d'accueil de différents groupes de réfugiés, essentiellement originaires du monde arabe. La part des réfugiés dans la population de la capitale syrienne est très importante puisqu'aux quelques centaines de Somaliens, Afghans, Soudanais ou Yéménites, il convient d'ajouter le groupe formé par les réfugiés palestiniens qui compte aujourd'hui 358603 personnes pour la seule agglomération damascène (sur un total de 456983 individus en Syrie) ${ }^{2}$ ainsi que l'importante population syrienne déplacée du Golan occupé, estimée à 305000 personnes ${ }^{3}$. Depuis 2003, plusieurs centaines de milliers de réfugiés irakiens s'y sont installés. La part de la population réfugiée et déplacée totale est donc très élevée pour une agglomération d'un peu plus de 4 millions d'habitants. 
3 Cet article vise à analyser la façon dont une population "déplacée" s'insère dans un environnement urbain et se recompose en exil, dans un contexte où la politique migratoire syrienne n'opère pas de distinction entre les réfugiés et les migrants. La Syrie, comme la majeure partie des Etats de la région, n'est ni signataire de la convention de Genève de 1951 ni des protocoles de New York de 1967 (Saleh, 2008 ; Zaiotti, 2006). Par ailleurs, il n'existe pas d'accords spécifiques régissant les relations entre le Haut Commissariat aux Réfugiés (HCR) et les autorités syriennes.La Syrie a maintenu jusqu'en octobre 2007 sa frontière ouverte aux ressortissants irakiens. Des tentatives pour limiter l'entrée d'Irakiens en Syrie ont été annoncées périodiquement. Durant le premier semestre 2007, la durée du visa accordé aux citoyens irakiens à la frontière a été modifiée à plusieurs reprises et réduite à un mois renouvelable (contre trois mois renouvelable précédemment). Depuis octobre 2007, les Irakiens doivent obtenir un visa préalablement à leur entrée en Syrie. Il existe deux principaux types de statut légal : le statut de "touriste", qui donne droit à séjourner la durée de validité du visa, et le statut de "résident temporaire" (carte de séjour d'un an), pour certaines catégories de personnes comme les parents d'enfants scolarisés en Syrie ou les personnes suivant un traitement médical et leur famille. De nombreuses personnes se retrouvent de facto en situation d'illégalité à expiration de leur visa de tourisme. Les autorités syriennes ont annoncé que la seule infraction au séjour ne constituerait pas un motif suffisant de reconduite à la frontière, annonce qui selon le HCR est respectée, les expulsions vers l'Irak étant limitées. Les Irakiens ne sont pas considérés par les autorités syriennes comme des réfugiés mais sont soumis à la législation et la réglementation sur l'entrée et le séjour des étrangers. De son côté le HCR délivre une lettre de protection temporaire (valable 1 an et renouvelable) aux réfugiés enregistrés auprès de ses services qui protège théoriquement du risque de déportation vers l'Irak. L'assistance aux réfugiés est délivrée par le HCR en collaboration avec le Croissant rouge syrien et des Organisations Non Gouvernementales syriennes et étrangères.

4 A partir d'un travail d'enquêtes auprès d'Irakiens vivant à Damas (débuté en février 2007) et à Stockholm (juin 2008), cet article propose d'analyser dans un premier temps l'installation des Irakiens dans l'agglomération damascène, puis de comprendre comment la capitale syrienne joue un rôle central dans l'organisation du champ migratoire irakien actuel.

\section{Les modes d'installation des Irakiens à Damas}

5 Les mouvements forcés de population, du fait de leur caractère le plus souvent massif, comme c'est le cas de l'Irak en proie à des conflits successifs depuis le début des années 1980, entraînent des transformations profondes des villes qui les accueillent. Les stratégies développées par les réfugiés en milieu urbain, inscrivent ces populations de façon durable dans le paysage des quartiers périphériques des grandes agglomérations du Sud. L'image du réfugié dépendant de l'aide humanitaire dans un camp isolé dans l'attente d'une solution venue de la communauté internationale est aujourd'hui à mettre en perspective avec celle plus nuancée du réfugié urbain, même si la distinction entre ces deux catégories est, elle, également sujette à caution, les trajectoires migratoires de nombreux réfugiés les faisant passer d'une catégorie à l'autre (Hyndman, 2000). 


\section{L'émergence des études sur les réfugiés urbains}

6 Depuis les années 1970, les études sur les populations réfugiées ont produit de très nombreuses catégories pour décrire leurs mouvements ainsi que leurs modes d'installations, telles que réfugié urbain, réfugié en camp, réfugié auto-installé, etc. (Black, 1991; Kunz, 1981; Rogge, 1977; Zetter, 2007). Ces dernières années, un nombre croissant d'études se sont intéressées à la question des réfugiés en milieu urbain, avec un accent particulier mis sur les problèmes de protection et d'accès aux services souvent mis en perspective avec la situation des camps de réfugiés (Agier, 2002; Al Qutub, 1989; Jacobsen, 2006; Malkki, 1995).

7 Les modes d'installation des populations réfugiées en ville sont traitées en tant que telles de façon croissante, de récentes études s'y sont intéressées au Moyen-Orient (Al Sharmani, 2003; Grabska, 2006; Le Houérou, 2007), en Afrique (Pérouse de Montclos, 2001 \& 2001a; Jacobsen, 2004) ou en Asie (Beaujard, 2008; Connór, 1989). Malgré la diversité des situations observées, ces études montrent que les mouvements de réfugiés s'inscrivent durablement dans leurs espaces d'accueil, et que la fin des conflits ou de la violence n'entraîne pas systématiquement le retour de l'ensemble de la population réfugiée. Les modes d'installation à l'œuvre s'avèrent donc pérennes et modifient en profondeur des quartiers entiers des villes du sud. Ainsi, les réfugiés ne sont pas simplement envisagés comme des récipiendaires de l'aide humanitaire, mais sont aussi considérés à travers leurs initiatives et les stratégies de survies et de solidarités qu'ils développent en exil. Les différentes situations étudiées, que ce soit au Caire, à Khartoum, Monrovia, New Delhi ou Peshawar, relèvent toutes l'importance des migrations forcées dans le développement urbain et leur articulation avec d'autres formes de migrations comme l'exode rural.

\section{Une forte présence irakienne en Syrie}

8 L'exode actuel des réfugiés irakiens a ses spécificités propres. Il n'y a pas eu d'exode massif en une ou deux vagues, comme cela a été le cas pour les Palestiniens en 1948 ou 1967, mais un flux croissant d'individus et de familles franchissant quotidiennement la frontière entre l'Irak et la Syrie. Depuis 2003, la Syrie a pratiqué une politique d'ouverture de sa frontière pour les Irakiens, considérés au même titre que les autres ressortissants arabes. Des mesures prises en septembre 2007, avec l'instauration d'un visa, restreignent de façon importante l'accès au territoire syrien pour les Irakiens. Les Irakiens ont accès au système d'éducation public syrien ainsi qu'au système de santé essentiellement par le biais du Croissant rouge syrien.

La présence irakienne en Syrie est assez ancienne, bien qu'elle n'ait jamais concerné un nombre aussi élevé de personnes qu'aujourd'hui. La Syrie a représenté tour à tour un espace d'accueil de réfugiés contraints de quitter leur pays pour des raisons politiques ou pour fuir les différents conflits que l'Irak a connus, mais aussi - et le plus souvent un espace de transit, la Syrie étant alors une porte de sortie vers les pays du bloc socialiste ou vers les pays occidentaux, ainsi qu'un espace d'accueil de migrants au sens plus large, mêlant commerce, travail et pèlerinage et cela bien que la frontière entre la Syrie et l'Irak ait été officiellement fermée entre 1982 et 1997 (Chatelard, 2005 : 134-137). 
Depuis les années 1970, la Syrie accueille des opposants au régime irakien. A partir de 1992, la situation change et la majeure partie des Irakiens qui se dirigent vers la Syrie s'exile en raison du contexte politique mais aussi des difficultés économiques de l'Irak. On assiste alors à l'arrivée de nombreux jeunes hommes qui s'installent en grand nombre à Sayda Zaynab, un quartier populaire de la banlieue sud de Damas et exercent toute une série de petits métiers, comme vendeurs de rue. Les plus pauvres sont restés en Syrie jusqu'en 2003, les plus riches ou ceux qui disposent de réseaux migratoires structurés ont réussi à s'exiler en Europe, en Amérique du nord ou en Australie; certains, enregistrés auprès $\mathrm{du} H C R$, ont pu bénéficier des programmes de réinstallation dans un pays tiers. Certains sont installés en Syrie, d'autres, comme les opposants politiques au régime de Saddam Hussein sont rentrés en Irak. Une diaspora irakienne se développe depuis plusieurs décennies constituée de différentes vagues de migrants et/ou de réfugiés (Al-Ali, 2007; Chatelard, 2005).

11 Si de nombreux Irakiens se sont installés ou ont transité par la Syrie depuis les années 1970, le conflit de 2003 représente un tournant par l'ampleur du nombre de réfugiés et de migrants qui ont franchi la frontière entre la Syrie et l'Irak. Le HCR estime qu'en 2003, avant le conflit en Irak, entre 60000 et 70000 Irakiens résidaient en Syrie ${ }^{4}$. Avec la chute du régime de Saddam Hussein une partie de ces réfugiés irakiens ont regagné l'Irak. Un mouvement de retour s'est donc amorcé et ce jusqu'en 2004, date à laquelle de nouveaux réfugiés sont venus trouver asile en Syrie. Les premiers arrivés étaient en général des personnes assez aisées qui pensaient s'installer pour une période limitée. Ils ont investi en Syrie en achetant des magasins et/ou des appartements. La plupart des arrivées récentes sont le fait d'Irakiens défavorisés, qui viennent sans capital économique et disposent de très faibles ressources.

Réfugiés irakiens enregistrés auprès de HCR -1993-déc. 2008

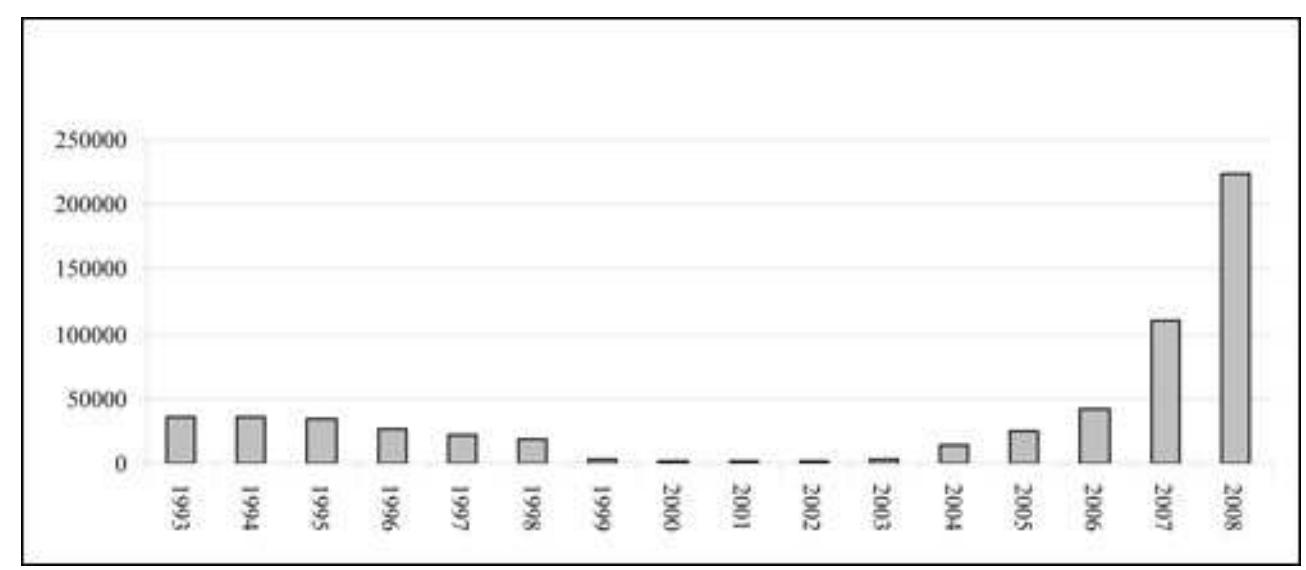

Source : UNHCR, 2008 \& UNHCR Syria Update, Damas, fév. 2009

La forte augmentation de la population irakienne en Syrie est lisible à travers la forte hausse du nombre de réfugiés enregistrés auprès du HCR, même si ce dernier demeure potentiellement faible au regard du nombre supposé de réfugiés irakiens présents sur le sol syrien selon les autorités syriennes. En l'absence de recensement de la population irakienne résidente en Syrie, et en raison de la forte diversité des situations migratoires existantes, il est difficile d'évaluer le nombre d'Irakiens présents actuellement sur le sol syrien $^{5}$ (ICG, 2008 : 16; Leenders, 2008 : 103). Les débats sur le nombre d'Irakiens en Jordanie montrent la difficulté de ce type d'exercice et les enjeux qu'il recouvre ${ }^{6}$. 
Comme l'indique Karen Jacobsen: "[t]he hidden, marginalized nature of urban refugees makes it difficult to make accurate estimates, and each 'authoritative' source has its own agenda and set of reasons for the number it puts out" (Jacobsen, 2006 : 275).

L'ampleur du phénomène migratoire peut cependant être saisie par l'augmentation du nombre d'Irakiens franchissant la frontière pour entrer en Syrie depuis 2000. Même si le nombre d'allers et retours augmente de façon très importante les chiffres, ces données montrent de façon très claire l'intensité croissante de la mobilité entre l'Irak et la Syrie, et l'augmentation induite du nombre d'Irakiens présents en Syrie. Ces chiffres révèlent donc une intense mobilité des Irakiens vers la Syrie jusqu'en 2007. Il faut retenir que le mouvement est de très forte ampleur numérique et induit d'importantes recompositions de la société irakienne en exil.

Nombre d'entrée de ressortissants irakiens en Syrie (1999-2007

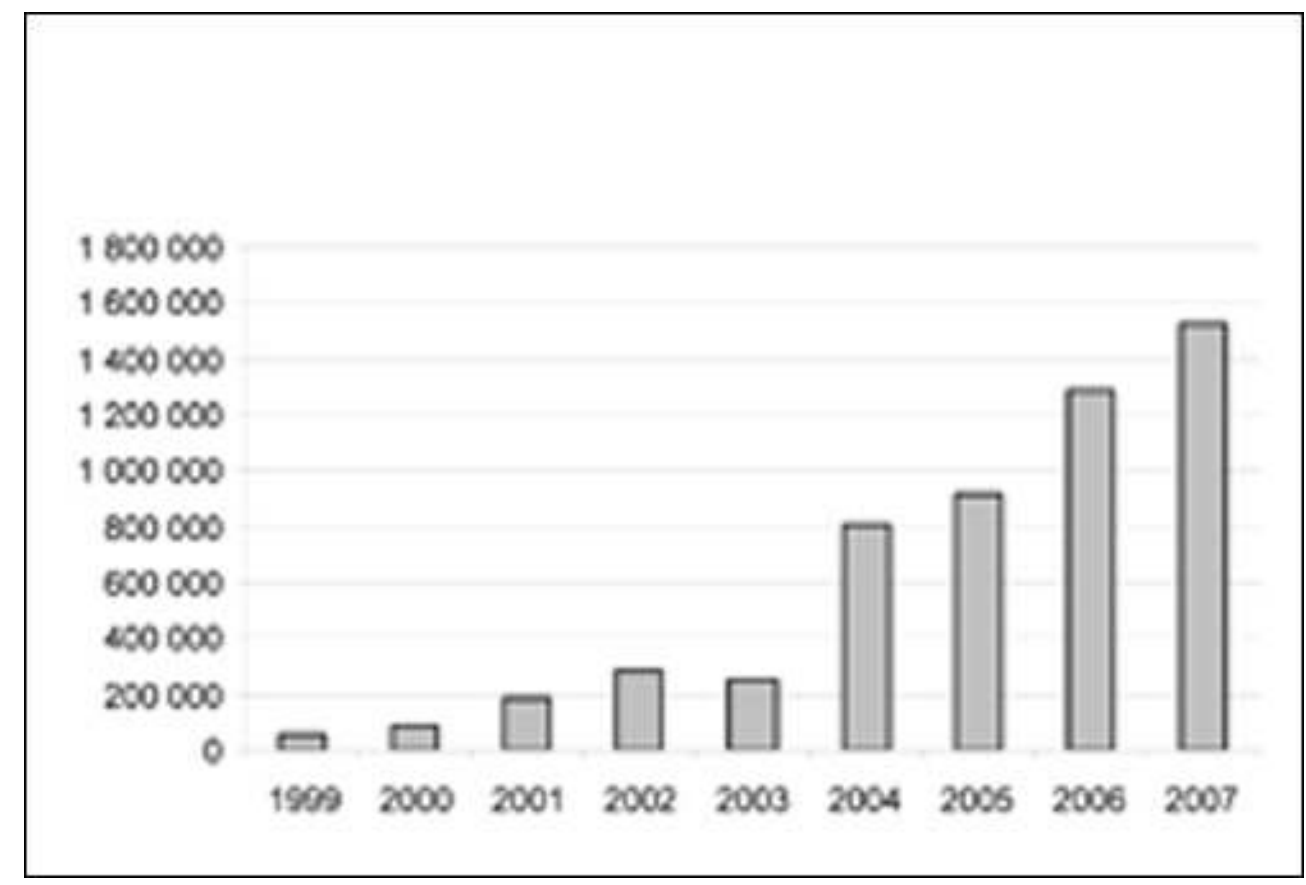

Source : Central Bureau of Statistics, Damas, 2008

Il n'existe cependant pas de recensement de la population irakienne résidente en Syrie. Le HCR a enregistré aujourd'hui plus de 224000 réfugiés, mais étant donnée la diversité des situations migratoires, la multiplicité des itinéraires et la très forte hétérogénéité de la population irakienne, nombre d'entre eux ne sont pas enregistrés auprès de l'organisation onusienne, comme c'est le cas d'autres réfugiés urbains à travers le monde (Jacobsen, 2004:60). Les autorités syriennes avancent le chiffre de plus d'un million d'Irakiens sur leur sol, mais cette estimation semble majorée en raison de la difficulté à enregistrer les sorties (Leenders, 2008 : 103). La forte disparité dans les chiffres est en partie le résultat de la difficile catégorisation de la population irakienne en Syrie. Alors que le HCR enregistre des "réfugiés", les autorités syriennes ne leur reconnaissent pas ce statut; elles comptabilisent les ressortissants irakiens. De nombreux irakiens résidents en Syrie ne se considèrent pas comme réfugiés, et/ou ne s'estiment pas dans le besoin d'assistance, ils ne s'inscrivent donc pas auprès du HCR. 


\section{Une population concentrée à Damas}

Plus des deux tiers de la population irakienne enregistrée auprès du HCR réside dans la région de Damas (Carte 1).

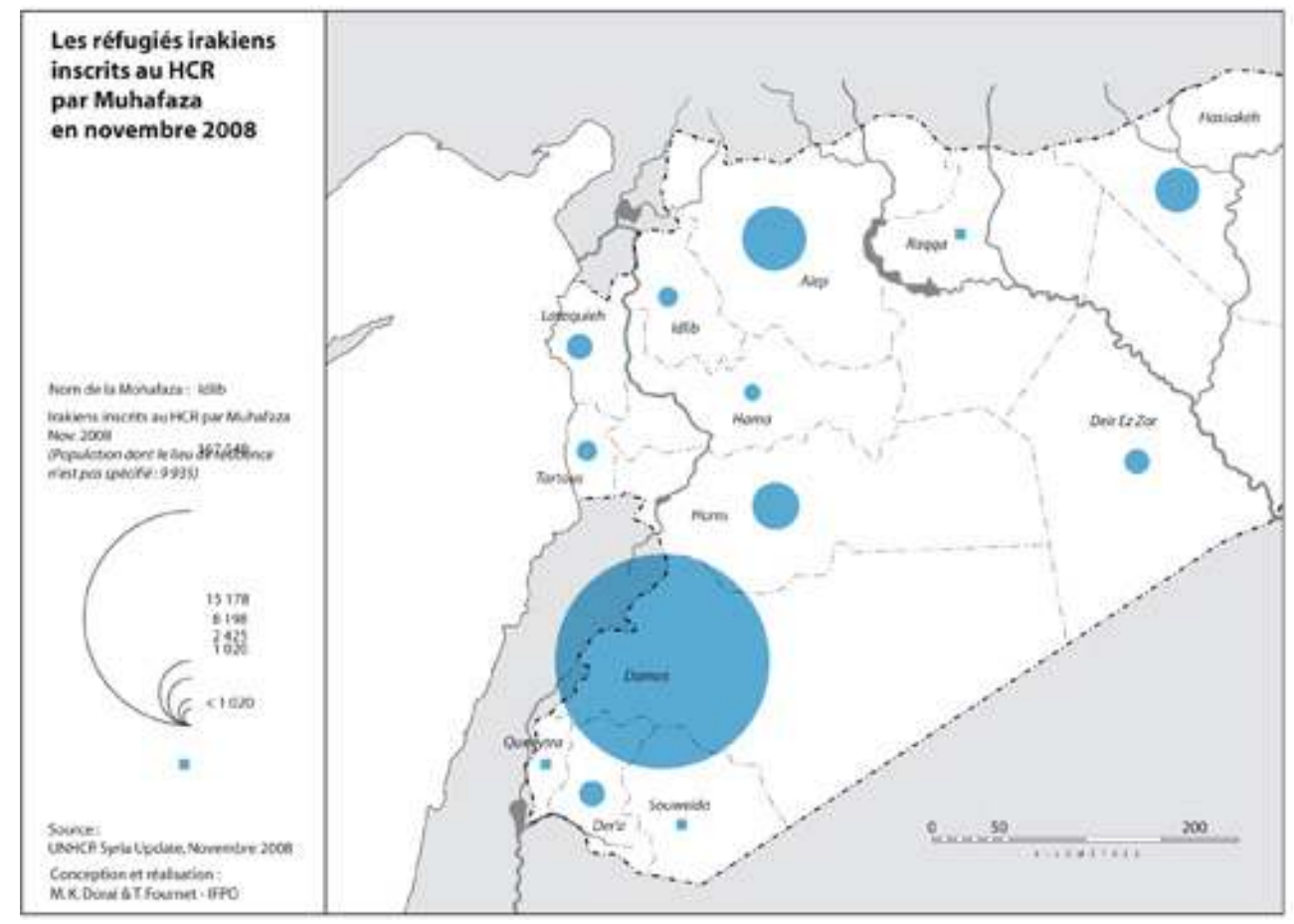

Les réfugiés, inscrits ou non auprès de l'organisation onusienne sont localisés dans les villes. Il n'existe pas de camps de réfugiés pour les héberger, celui qui existait à Hassakeh proche de la frontière syro irakienne a fermé ses portes en juin 2004. Les Irakiens présents en Syrie sont majoritairement d'origine urbaine, Bagdad étant la principale ville touchée par l'exode actuel. Les Irakiens se sont donc dirigés vers la capitale syrienne, où les précédentes vagues migratoires s'étaient installées, mais aussi parce qu'il est plus facile d'y trouver un emploi et également en raison de la proximité des agences internationales (Haut Commissariat aux Réfugiés, Organisation Internationale des Migrations, associations caritatives locales et internationales) et des ambassades étrangères. Les réfugiés palestiniens d'Irak forment une catégorie à part et près de $2500 \mathrm{~d}$ 'entre eux se trouvent dans trois camps, un en Syrie ( $\mathrm{Al} \mathrm{Hol} \mathrm{dans} \mathrm{le}$ gouvernorat de Hassakeh - 334 réfugiés), un en Irak (Al Walid - 1367 réfugiés) et un dans le no man's land entre les deux pays ( $\mathrm{Al}$ Tanf -830 réfugiés) ${ }^{7}$. 


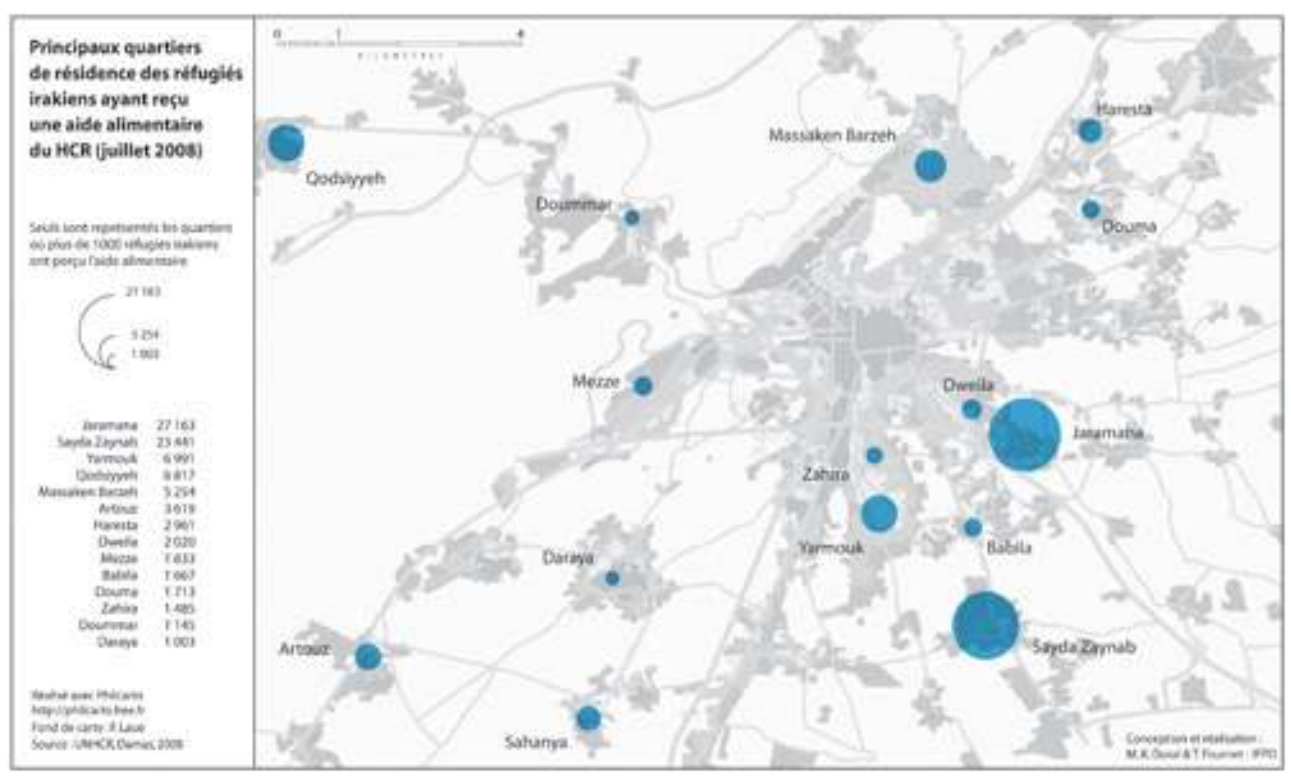

17 Comme le montre la carte 2, la présence irakienne à Damas se polarise dans les quartiers de la périphérie de Damas comme Sayda Zaynab, Jaramana, Massaken Barzeh, Yarmouk ou Qodsiyyeh et de plus en plus dans des espaces plus périphériques où le prix des logements est moins élevé comme Sednaya ou Tell, et dans une moindre mesure dans d'autres villes comme Alep, Lattaquieh ou Deir el Zor.

La répartition spatiale est le résultat de trois phénomènes complémentaires. D'un côté, les Irakiens se répartissent en fonction de leurs capacités financières, les plus pauvres se concentrant dans les quartiers les plus défavorisés. De l'autre, on assiste à des formes de polarisation liées aux vagues migratoires précédentes et à des formes de regroupement communautaire dues à la présence d'agences caritatives ou d'institutions religieuses venant en aide aux réfugiés. Enfin, les Irakiens se sont dirigés vers des espaces où s'étaient installés ou bien avaient transité leurs coreligionnaires avant 2003. Les réfugiés irakiens se sont installés dans des quartiers périphériques de l'agglomération damascène dans des espaces où la présence migrante est forte depuis la fin des années 1940. On retrouve effectivement dans ces quartiers des réfugiés palestiniens installés depuis 1948 ou 1967, des déplacés syriens originaires du Golan, ainsi que des migrants internes venus des zones rurales. On peut y ajouter les réfugiés somaliens ou les migrants soudanais qui sont installés dans ces mêmes espaces. Damas polarise les migrations internes syriennes, et aujourd'hui un cinquième de la population de l'agglomération est née ailleurs, cette proportion n'incluant pas les Irakiens (Al Baridi, 2005 : 125; Khawaja, 2002 : 26-28).

Les quartiers habités par les Irakiens changent de physionomie depuis leur arrivée dans la capitale syrienne, preuve s'il en est que le "transit" de ces migrants se prolonge suffisamment pour qu'il s'inscrive dans le paysage urbain. Les commerces et restaurants irakiens ainsi que les agences de voyage spécialisées sur l'Irak se développent dans la périphérie damascène et modifient localement le paysage urbain comme c'est le cas à Jaramana ou à Sayda Zaynab. 


\section{L'inscription des Irakiens dans le paysage urbain damascène}

\section{types d'activités, qui vont du vendeur de rue, à l'atelier de confection en passant par la} petite épicerie de quartier. Ces modes d'installation sont liés tant aux parcours personnels des entrepreneurs qu'aux conditions d'accueil en milieu urbain, qui ont favorisé la mise en place de stratégies d'adaptation à leur nouvel environnement assez souples. La diversité des situations migratoires - réfugiés fuyant des persécutions, personnes fuyant l'insécurité généralisée, commerçants ou entrepreneurs dans l'impossibilité de travailler, etc.-, et l'accueil réservé aux Irakiens en Syrie, ont permis à la société irakienne de se recomposer en exil et de s'adapter à la société d'accueil, même si l'exil forcé a généré une dégradation générale du niveau de vie des Irakiens et des tensions accrues dans la société syrienne dues à l'importance de la présence irakienne dans certains quartiers.

21 A Sayda Zaynab, à l'ouest du rond point de Hajjira s'étend le quartier où résident un nombre très important d'Irakiens, son artère principale est d'ailleurs surnommée "rue des Irakiens" et de très nombreux commerces s'y sont développés. Le long de cet axe principal qui irrigue des ruelles perpendiculaires, de nombreuses activités liées à la présence irakienne se sont développées. On peut mentionner dans un premier temps les agences de transport -très présentes également à Jaramana- qui vendent des places de taxi ou de bus vers les principales villes irakiennes. Des conducteurs de taxi irakiens proposent leurs services à ces agences et transportent passagers, valises et cartons de marchandises à destination de l'Irak. L'instauration du visa préalablement à l'entrée en Syrie a entrâné une forte diminution du trafic des taxis et des bus. Le renouvellement du visa d'entrée à la frontière syro-irakienne tous les 6 mois représentait une des activités importantes de ces agences.

De très nombreux petits commerces de proximité se sont implantés tant à Jaramana qu'à Sayda Zaynab: des restaurants populaires proposant des plats traditionnels irakiens, du pain irakien, des petites épiceries qui importent des denrées alimentaires irakiennes, auparavant non disponibles en Syrie. Des vendeurs de rue proposent sur leur petite carriole des pâtisseries irakiennes à emporter, de la carpe pour la préparation du Masgouf, ou bien du thé irakien. Des échoppes plus importantes se sont également développées. On peut citer par exemple l'installation de la pâtisserie $\mathrm{Al}$ Baghdadi à Sayda Zaynab qui produit de nombreux types de pâtisserie irakienne qui se vendent ensuite dans les autres quartiers irakiens de Damas. A Jaramana on retrouve par exemple aujourd'hui plusieurs restaurants irakiens sur la principale rue d'entrée dans le quartier lorsque l'on arrive de Damas, et cette activité est en pleine expansion. Dans les ruelles plus étroites de ces deux quartiers on retrouve de nombreux petits commerces qui proposent des vêtements traditionnels irakiens, ainsi que des écharpes, bonnets, drapeaux marqués aux couleurs de l'Irak. Les maillots de l'équipe de football irakienne vainqueur de la coupe d'Asie des nations en 2007 sont très présents.

\section{Damas, une centralité migratoire irakienne}

Les réfugiés appartiennent à des groupes socio-économiques ou confessionnels plus ou moins organisés, plus ou moins importants numériquement et qui disposent de relais ou non dans la diaspora constituée depuis les années 1970. Les familles sont le plus 
souvent dispersées entre plusieurs Etats, et si les réseaux de solidarité tendent à se reconstruire dans l'exil, le caractère récent et massif de l'exode actuel tend à rendre difficiles ces connexions. L'écart se creuse dans l'exil entre les familles avec d'importantes connexions locales et/ou transnationales et celles qui ont un accès limité aux ressources et à la mobilité. Les modes d'installation et les regroupements qui se sont opérés permettent cependant à Damas de jouer un rôle central dans l'organisation des différentes formes de mobilités irakiennes.

\section{De l'exode à l'exil : parcours migratoires complexes et logiques plurielles}

24 La Syrie est l'un des seuls pays à accueillir encore aujourd'hui les Irakiens sur son sol, même si elle a adopté une politique plus restrictive depuis la fin 2007. Le pays est à la fois un espace refuge, un espace de transit mais aussi un espace d'installation sur le moyen terme. L'émigration irakienne ne s'est pas produite en une ou deux vagues principales, mais s'est construite sur la durée, avec des temps d'exil ponctués parfois de retours puis de départs définitifs.

25 A cause de l'insécurité, la famille a dû quitter Mossoul en 2004 pour se réfugier à Qamichli [une ville syrienne proche de la frontière irakienne]. Ils y sont restés 6 mois, pendant lesquels le père faisait des allers-retours pour continuer à faire fonctionner son entreprise du bâtiment. La famille est retournée vivre à Mossoul au début de l'année 2005, la situation sécuritaire s'étant améliorée. En février 2006, ils ont été contraints de partir à nouveau, définitivement cette fois, et ils se sont installés dans un village près de Damas. Avec l'argent qu'ils avaient amené d'Irak, après avoir tout vendu, le père et son épouse ont émigré illégalement en Suède en juillet 2006. Leurs deux fils majeurs ne peuvent prétendre au regroupement familial, ils ont essayé de les rejoindre à deux reprises avec des passeurs, mais ils ont échoué et ont perdu à chaque fois beaucoup d'argent.

Ce récit d'un petit entrepreneur irakien qui réside depuis peu en Suède n'est pas isolé. Nombre d'Irakiens ont quitté leur pays dans l'espoir de pouvoir y revenir rapidement et ont tenté d'y poursuivre quand cela est possible une activité économique en se rendant le plus souvent possible en Irak. La frontière avec la Syrie étant ouverte, les allers-retours sont nombreux. Ce n'est que lorsque la situation en Irak s'est dégradée de façon significative, souvent dans le courant de l'année 2006, que des familles entières ont décidé de s'exiler définitivement, certains tentant leur chance vers l'Europe. Si, pour quelques milliers d'Irakiens, la Syrie n'est qu'une étape dans un parcours qui les mènera vers l'Europe, les Etats-Unis ou l'Australie, elle est pour la majeure partie d'entre eux un espace refuge, où ils peuvent retrouver la sécurité et tenter de renouer avec une vie "normale" en exil.

Pour d'autres, Damas pensée dans un premier temps comme une simple étape, devient leur espace d'installation dans la durée. Si l'exil leur a apporté la sécurité et la possibilité de retrouver une vie normale, il s'est souvent accompagné d'un déclassement social source de précarité, comme en témoigne le récit d'Abou Jaber. Il réside actuellement dans un quartier de la banlieue de Damas.

28 Abou Jaber est un couturier de 57 ans qui a quitté Bagdad fin juillet 2006 pour des raisons de sécurité. Il réside actuellement à Sayda Zaynab avec son 
épouse et ses quatre enfants. L'un de ses fils travaillait comme chauffeur au ministère du pétrole, ce qui lui a valu des menaces après 2003 en tant que fonctionnaire de l'Etat. A Bagdad, il était à son compte et disposait d'un atelier de couture avec 20 machines à coudre. Le conflit lui a fait perdre son outil de travail. Ayant dû quitter précipitamment Bagdad, il n'a pu vendre qu'une partie de ses biens et est arrivé avec la somme de 4000 USD. Il a opté pour la Syrie après avoir longtemps hésité avec la Jordanie, mais suite aux différentes restrictions imposées par le royaume Hachémite - et bien qu'il connaisse des proches qui s'y sont installés - il a préféré la Syrie où les conditions d'accueil sont meilleures. De plus, ses activités en Irak l'avaient amené à connaître la Syrie où il achetait des tissus depuis les années 1970. Arrivé à Damas par le bus il s'est rapidement dirigé vers Lattaquié où le prix des appartements à louer étaitinférieur. L'invasion par Israël du Liban en juillet 2006 a entraîné la venue de plusieurs dizaines de milliers de réfugiés qui se sont installés pour partie à Damas ce qui a entraîné une importante pression sur le marché locatif. Il n'est cependant resté qu'un mois à Lattaquié faute de pouvoir trouver un travail, et du fait de l'absence d'une communauté irakienne. Il s'est donc dirigé vers Sayda Zaynab, où il loue un appartement pour 20000 LS (400 USD) pour 2 chambres et un salon. Après une période de chômage et de petits emplois précaires, il a trouvé à louer un petit atelier de couture qui dispose d'une seule machine à coudre sur laquelle il travaille. Deux de ses fils l'aident. A eux trois ils arrivent à gagner 500 LS par jour (10 USD).

Un entrepreneur irakien a développé depuis 2006 ses activités qu'il a transférées de Bagdad vers Jaramana. Son itinéraire personnel montre la pluralité des logiques à l'œuvre dans l'émigration actuelle des Irakiens vers la Syrie. Son installation en Syrie relève, d'un côté de la très forte dégradation de la situation sécuritaire en Irak, et de l'autre d'une logique de sécurisation et de développement de ses activités économiques. Il est effectivement le seul entrepreneur de sa famille, mais aussi le seul, dans sa famille proche, à avoir quitté l'Irak.

30 Un jeune entrepreneur irakien se spécialise dans la confection des abayas à Bagdad [tenue vestimentaire qui se présente sous la forme d'un long manteau]. Il développe son activité et ouvre un atelier de confection en 1998 et plus tard un magasin de prêt-à-porter. Il agrandit son atelier et en 2000, il emploie six salariés. Alors que la concurrence se développe, il voyage à l'étranger pour trouver de nouveaux modèles. Il se met alors à aller régulièrement à Alep (une semaine par mois) pour acheter des vêtements en gros qu'il expédie dans son magasin à Bagdad et des accessoires en gros pour son atelier de confection à Bagdad. En discutant avec les grossistes d'Alep il apprend que les ateliers de production d'accessoires pour le textile (perles, paillettes, bijoux, etc.) sont produits dans le quartier de Hariqa dans la vieille ville de Damas. Il se rend donc sur place pour acheter en gros des accessoires qu'il envoie dans ses ateliers de production à Bagdad. Il effectue donc des allers et retours mensuels entre Bagdad, où il vit et détient son magasin et son atelier, et Alep et Damas où il se fournit en vêtements, tissus et accessoires en gros. En octobre 2006, il ferme son usine et son magasin à Bagdad à cause des nombreuses coupures d'électricité qui occasionnent des retards dans son atelier et en raison de la situation sécuritaire qui s'est 
fortement dégradée et l'empêche de circuler dans Bagdad et vers la Syrie. Il décide alors de s'installer à Jaramana pour plusieurs raisons : de nombreux entrepreneurs irakiens s'y sont préalablement installés, de nombreuses entreprises de transport qui travaillent avec l'Irak sont installées dans ce quartier, c'est un lieu de vente en gros pour le textile, et on trouve facilement de la main d'œuvre spécialisée. Il emploie 13 ouvriers et ouvrières syriens et irakiens. Sa production ( 120 pièces par jour) est intégralement expédiée et vendue en Irak à ses anciens clients. Son installation en Syrie lui offre plusieurs avantages comme la sécurité et la stabilité de l'activité, la proximité avec les grossistes et la possibilité de voyager pour suivre les dernières innovations dans la mode. Il s'est par exemple récemment rendu en Chine pour y acheter des nouveaux accessoires non encore disponibles sur le marché syrien. Toute sa famille (frères, sœurs, parents) est restée vivre à Bagdad. Il travaille d'ailleurs avec un de ses frères resté sur place qui s'occupe de ses affaires, des discussions avec les clients et de l'envoi d'argent. moins longues, et leurs logiques plurielles (asile, refuge temporaire, transit, commerce, pèlerinage) sont des éléments qui caractérisent aujourd'hui la migration irakienne en Syrie. La majeure partie des Irakiens peuvent être considérés comme réfugiés, mais l'absence de reconnaissance de ce statut par les autorités syriennes, rend leur installation précaire et réversible. Si les entrepreneurs ont réussi à stabiliser leur situation socioprofessionnelle dans leur pays d'accueil, beaucoup d'Irakiens connaissent une baisse importante de leur niveau de vie, et ne peuvent trouver du travail. Nombreux sont donc ceux qui cherchent un nouveau pays d'accueil pour s'installer durablement, et tentent d'émigrer de façon légale ou illégale.

A la suite de la guerre du Golfe de 1991, la Jordanie a joué le rôle de porte de sortie d'Irak et d'espace de transit pour plus d'un demi-million d'Irakiens qui ont trouvé refuge à travers le monde (Chatelard, 2002). Aujourd'hui, la Syrie joue également ce rôle de centralité migratoire du fait de sa politique migratoire souple, même si des restrictions à l'entrée et au séjour ont été mises en place en octobre 2007. Les relations avec l'Irak demeurent fortes, et les taxis, bus ou camions faisant l'aller-retour sont nombreux au départ de la capitale syrienne et d'Alep. Selon les autorités syriennes citées par le HCR, il y aurait quotidiennement 500 entrées par la frontière d'Al Tanf, un chiffre équivalent aux sorties. La dernière poussée de violence à l'automne 2008 à l'encontre des Irakiens de confession chrétienne à Mossoul a vu l'arrivée de plusieurs centaines de familles qui ont trouvé refuge en Syrie.

Aujourd'hui, de nombreuses familles sont divisées et résident dans plusieurs pays d'exil au Moyen-Orient et au-delà. La période passée à Damas ne constitue pas un simple temp d'attente, mais joue un rôle important dans l'élaboration du projet migratoire. L'ensemble des membres d'une même famille le plus souvent ne quittant pas l'Irak à la même date, le regroupement familial s'effectue à Damas, ce qui permet d'activer les réseaux de solidarité et de faciliter l'installation dans le pays d'accueil. Les derniers arrivants bénéficient d'un logement qu'ils partagent avec le reste de leur famille et éprouvent moins de difficultés à trouver de l'emploi. Ils vivent dans l'attente d'un départ prochain vers un pays tiers qui les acceptera, sans avoir cependant de certitude sur cet avenir, le nombre de réinstallations par le HCR demeurant très faible (un peu 
moins de 8000 en 2008) ${ }^{8}$ faute de trouver des pays d'accueil. La Syrie est également un lieu de repli pour des Irakiens déboutés du droit d'asile dans les pays développés et qui, reconduits en Irak, sont contraints de s'exiler à nouveau en Syrie dans l'attente d'un nouveau départ. Face à l'instabilité qui perdure en Irak et à la fermeture des portes de nombreux pays aux demandeurs d'asile (Sperl, 2007) et donc à la difficulté pour les Irakiens à voyager, les regroupements familiaux se déroulent souvent à Damas, où se multiplient les mariages avec des Irakiens de la diaspora venus d'Australie, du Canada, des Etats-Unis, de Suède ou d'Allemagne. Leurs conjoints ou conjointes les rejoignent ensuite par le biais du regroupement familial.

La Syrie est également le point de passage obligé pour ceux qui veulent se rendre au Liban qui accueille aujourd'hui une cinquantaine de milliers d'Irakiens sur son sol. Ceux qui ont obtenu leur carte de séjour au Liban se rendent à Damas pour y retrouver l'ambiance irakienne et participer à des fêtes comme l'achoura pour les musulmans chiites qui se déroule à Sayda Zaynab. Ils peuvent aussi trouver de nombreux produits irakiens importés et vendus dans des petites échoppes, ce type de commerce ne s'étant pas du tout développer au Liban, où la communauté irakienne a du mal à s'afficher dans l'espace public.

\section{Conclusion.}

Les conséquences de l'arrivée massive de réfugiés irakiens en Syrie sont multiples et ont soulevé de nombreux débats quant à la pression sur le marché locatif, l'augmentation des prix, la dégradation de la sécurité dans certains quartiers, la concurrence sur le marché de l'emploi, etc. sans que le rôle de la population réfugiée dans ces processus ne soit évalué de façon spécifique. La Syrie ne fait pas ici figure d'exception, l'arrivée de réfugiés entraîne souvent ce type de polémique (Hyndman, 2000 : 160-162). L'accueil et l'installation sur le moyen terme des Irakiens a également contribué à s'interroger tant sur l'évolution de leur statut juridique que de leur rôle supposé - dans la géopolitique régionale (Leenders, 2008a).

La crise irakienne, déclenchée en 2003, est à replacer dans le contexte plus large des guerres qui l'ont précédée, de la dégradation de la situation économique liée à l'embargo et de la répression des mouvements d'opposition au régime irakien. L'émigration actuelle, si elle se singularise par son ampleur, s'inscrit dans un continuum avec les précédentes vagues d'émigration ${ }^{9}$. On assiste donc au développement de pôles d'ancrages dans les quartiers périphériques de Damas qui peuvent remonter pour certains - comme Sayda Zaynab - aux années 1970. Dans les faits, les formes d'intégration locale s'articulent le plus souvent avec des connections transnationales, et représentent donc une étape dans le parcours migratoire soit vers un pays tiers, soit pour sécuriser et préparer un éventuel retour. La question de la présence des réfugiés irakiens en milieu urbain, de leurs modes de regroupement et de leurs pratiques de l'espace constitue un apport à la réflexion sur les catégories de réfugié et migrant urbains, et sur les formes d'organisation de l'espace qui découlent de leur présence dans leurs pays d'accueil.

L'installation des Irakiens en milieu urbain de leur propre initiative a permis à ces derniers de recomposer leurs réseaux socio-communautaires et de développer des activités économiques propres en s'insérant dans le tissu urbain damascène. Une politique de mise en place de camps d'accueil aurait certainement eu pour effet de 
développer la dépendance des réfugiés envers la communauté internationale et une intégration beaucoup plus limitée dans le milieu environnant. L'auto-installation des réfugiés a permis à ces derniers d'initier leurs propres stratégies de survie et de s'adapter à leur nouvel environnement, même si la durée de l'exil entraîne l'augmentation forte de la dépendance des Irakiens les plus défavorisés et ne bénéficiant pas de soutiens à l'étranger envers les organisations internationales

\section{BIBLIOGRAPHIE}

Agier M., 2002. "Between War and City: Towards an Urban Anthropology of Refugee Camps", Ethnography, vol. 3, $\mathrm{n}^{\circ} 3: 317-341$.

Al-Ali N., 2007. "Iraqi Women in Diasporic Spaces: Political Mobilization, Gender and Citizenship", Revue des mondes musulmans et de la Méditerranée, $\mathrm{n}^{\circ}$ 117-118; 137-153.

Al-Ali N., 2004. The Relationship between Migration within and from the Middle East and North-Africa and Pro-Poor Policies, A Report by the Institute of Arab \& Islamic Studies, University of Exeter for the Department for International Development, $48 \mathrm{p}$.

Al-Qutub I. Y., 1989. "Refugee Camp Cities in the Middle East: A Challenge for Urban Development Policies", International Sociology, vol. 4, n 1 : 91-108.

Al-Sharmani M., 2003. "Livelihood and Identity Constructions of Somali Refugees in Cairo", The American University in Cairo - Forced Migration and Refugee Studies, Working Paper $n^{\circ} 2,37 \mathrm{p}$.

Al Baridi O., 2005. Périurbanisation des métropoles arabes. La densification de cette couronne et la consommation des terres agricoles, Thèse de doctorat de l'Université de Paris XII, sous la direction de J. Steinberg, $231 \mathrm{p}$.

Beaujard J.,2008, Identité "réfugié", identité transversale. Les réfugiés à Delhi au sein des dynamiques institutionnelles, communautaires et associatives, Thèse de doctorat de l'Université d'Aix Marseilles I, sous la direction de F. Robinne, $502 \mathrm{p}$.

Black R., 1991. "Refugees and Displaced Persons: Geographical Perspectives and Research Directions", Progress in Human Geography, 15-3 : 281-298.

Chatelard G., 2005. "L'émigration des Irakiens de la guerre du Golfe à la guerre d'Irak

(1990-2003)", in H. Jaber et F. Métral (dirs.), Mondes en mouvements. Migrants et migrations au Moyen-Orient au tournant du XXIe siècle, Beyrouth, IFPO : 113-155.

Chatelard G., 2002. Incentives to Transit : Policy Responses to Influxes of Iraqi Forced Migrants in Jordan, EUI Working Papers, Florence : European University Institute RSC $n^{\circ} 2002 / 50$ - Mediterranean Programme Series, $29 \mathrm{p}$.

Connór K. M., 1989. "Factors in the Residential Choices of Self-Settled Afghan Refugees in Peshawar, Pakistan", International Migration Review, vol. 23, nº 4 : 904-932.

Grabska K., 2006. "Marginalization in Urban Spaces of the Global South: Urban Refugees in Cairo", Journal of Refugee Studies, vol. 19, $\mathrm{n}^{\circ}$ 3: 287-307. 
Hyndman J., 2000. Managing Displacement : Refugees and the Politics of Humanitarianism, Minneapolis : University of Minnesota Press (Coll. Borderlines, 16), $253 \mathrm{p}$.

International Crisis Group, 2008. Failed Responsibility: Iraqi Refugees in Syria, Jordan and Lebanon, Middle East Report n 77, p. 16. [http://www.crisisgroup.org/home/index.cfm?id=5563\&l=1]

Jacobsen K., 2006. "Editorial Introduction. Refugees and Asylum Seekers in Urban Areas: A livelihoods Perspective", Journal of Refugee Studies, vol. 19, $n^{\circ} 3: 275$.

Jacobsen K., 2004. "Just Enough for the City: Urban Refugees Make their Own Way", in World Refugee Survey, U.S. Committee for Refugees and Immigrants. [http://www.refugees.org/data/wrs/04/pdf/57-65.pdf]

Khawaja M., 2002. Internal migration in Syria. Findings from a National Survey, Oslo : FAFO Report 375, 140 p. [http://www.fafo.no/pub/rapp/375/index.htm]

Kunz E. F., 1981. "Exile and Resettlement: Refugee Theory", International Migration Review, vol. 15 : 42-51.

Leenders R., 2008. "L'adieu aux armes : la politique des réfugiés irakiens et son impact sécuritaire sur la région", Maghreb-Machrek, n¹98 : 93-122.

Leenders R., 2008a. "Iraqi Refugees in Syria: causing a spillover of the Iraqi conflict?", Third World Quarterly, vol. 29, $\mathrm{n}^{\circ} 8: 1563-1584$.

Le Houérou F., 2007. "Voisins ou ennemis à Arba Wa Nus? La mise en scène du quotidien des migrants forcés égyptiens et réfugiés sud soudanais dans un quartier populaire du Caire", Revue des mondes musulmans et de la Méditerranée, $\mathrm{n}^{\circ} 119-120: 59-80$.

Malkki L., 1995. Purity and Exile: Violence, Memory, and National Cosmology Among Hutu Refugees in Tanzania. Chicago, IL: University of Chicago Press.

Pérouse de Montclos M.-A., 2001. "Déplacés, insertion urbaine et modes de mobilisation : L'exemple de Monrovia en guerre", Canadian Journal of African Studies / Revue Canadienne des Études Africaines, vol. $35, \mathrm{n}^{\circ} 3: 518-544$

Pérouse de Montclos M.-A., 2001a. "Migrations forcées et urbanisation : le cas de Khartoum", Cahiers du CEPED, $\mathrm{n}^{\circ} 63,60 \mathrm{p}$.

Rogge J. R., 1977. "A Geography of Refugees: some Illustrations from Africa", Professional Geographer, $29: 186-193$.

Rossi S., 2008. "Le drame ignoré des Palestiniens d'Irak et l'exode vers la Syrie", Asylon(s), n 5 [http://www.reseau-terra.eu/article808.html]

Saleh, F., 2008, "La migration irrégulière en droit syrien", CARIM notes d'analyse et de synthèse 2008/56 - Série sur la migration irrégulière, module juridique, Institut Universitaire Européen, RSCAS, 7 p. [www.carim.org].

Sperl M., 2007. Fortress Europe and the Iraqi 'intruders': Iraqi asylum-seekers and the EU, 2003-2007, New issues in Refugee Research, Research Paper n 144, Genève : UNHCR, 19 p.

Zaiotti, R., 2006, "Dealing with Non-Palestinian Refugees in the Middle East: Policies and Practices in an Uncertain Environment”, International Journal of Refugee Law, vol. 18, n 2 : 333-353.

Zetter R., 2007. "More Labels, Fewer Refugees: Remaking the Refugee Label in an Era of Globalization", Journal of Refugee Studies, vol. 20, nº 2 : 172-192. 


\section{NOTES}

1. UNHCR Statistical Yearbook, 2005: 55.

2. Source : UNRWA, Juin 2008 [ http://www.un.org/unrwa/publications/pdf/ rr_countryandarea.pdf

3. Cf. Syria: Forty years on, people displaced from the Golan remain in waiting, Internal Displacement Monitoring Centre (IDMC) - Norwegian Refugee Council, 31 octobre 2007 [www.internal-displacement.org]

4. "Some 2,400 Iraqis have been granted or are being considered for refugee status by UNHCR. Furthermore, there are around 60,000-70,000 Iraqis who have never approached UNHCR or have been denied refugee status through the UNHCR refugee status determination process and continue to reside illegally in the country, tolerated by the Syrian authorities", UNHCR, Preliminary Repatriation and Reintegration Plan for Iraq, 30 April 2003 (www.reliefweb.int)

5. Pour un aperçu de la teneur du débat sur les chiffres en Syrie on peut se reporter au rapport de l'International Crisis Group, Failed Responsibility: Iraqi Refugees in Syria, Jordan and Lebanon, Middle East Report $\mathrm{N}^{\circ} 77$ - 10 Juillet 2008, p. 16. [http://www.crisisgroup.org/home/index.cfm? id=5563\&l=1]

6. Iraqis in Jordan 2007 - Their Number and Characteristics, FAFO - Oslo, Norvège [http:// www.fafo.no/ais/middeast/jordan/IJ.pdf]

7. Ces derniers sont contraints de quitter l'Irak suite aux violences particulières qu'ils subissent et se voient dénier pour la plupart l'entrée dans les pays arabes qui pourraient constituer selon ces pays un préalable à leur réinstallation, remettant en cause leur droit au retour (Cf. Ruppert Colville, "Shame. How the world has turned its back on the Palestinian refugees in Iraq", Refugees, $\mathrm{n}^{\circ}$ 142, issue 2, $2007: 24$ ). Quelques centaines de réfugiés palestiniens d'Irak ont cependant été admis à entrer en Syrie (Rossi, 2008). Les chiffres cités sont extraits de UNHCR Syria Update, Damas, fév. 2009.

8. UNHCR Syria Update, fév. 2009 : 7.

9. Cf. G. Chatelard "Emigrating from Iraq in the period 1991-2007: social networks as alternatives for international protection", présenté à Annual conference of the International Association for Contemporary Iraqi Studies (IACIS), Philadelphia University, Amman [http:// hal.archives-ouvertes.fr/hal-00338438/fr/]

\section{RÉSUMÉS}

Depuis la chute du régime de Saddam Hussein, le conflit en Irak perdure et des centaines de milliers d'Irakiens ont été contraints de quitter leur pays pour se diriger en grand nombre vers la Syrie. Pour la plupart venus des principales agglomérations irakiennes, et surtout de Bagdad, ils se sont installés dans les faubourgs de la capitale syrienne. Ni la Syrie, ni le Haut Commissariat aux Réfugiés des Nations Unies n'ont ouverts de camps pour accueillir les réfugiés, qui forment aujourd'hui l'une des plus importantes populations de réfugiés urbains à travers le monde. Cet article analyse les modes d'installation des Irakiens à Damas, et le rôle que joue la ville dans les parcours migratoires des exilés.

Since the fall of Saddam Hussein regime, the conflict in Iraq is still ongoing and hundreds of thousands of Iraqis have been constrained to leave their country to move in great number 
towards Syria. The vast majority comes from the main cities in Iraq, and especially from Baghdad, and they settled in the suburbs of the Syrian capital. Neither Syria, nor the United Nations High Commissioner for Refugees have opened camps to accommodate the refugees, who are today one of the most important group of urban refugees throughout the world. This article analyzes the modes of settlement of the Iraqis in Damascus, and the role played by the city in the migratory dynamics of the Iraqi Diaspora.

\section{INDEX}

Mots-clés : Irakiens, Damas, Syrie, Réfugiés urbains, Diaspora

Keywords : Iraqis, Damascus, Syria, Urban refugees, Diaspora

\section{AUTEUR}

\section{MOHAMED KAMEL DORAÏ}

Mohamed Kamel Doraï (Mohamed-Kamel.Dorai@univ-poitiers.fr) est géographe, chargé de recherches au CNRS à l'Institut Français du Proche-Orient de Damas (Syrie). Il a récemment publié :

- Doraï M. K., Puig N. Dirs., 2008. "Les Palestiniens en / hors camps. Formes sociales, Pratiques des interstices", Asylon(s), n 5, [http://terra.rezo.net/rubrique146.html]

- Doraï M. K., 2008. "Du camp à la ville. Migrations, mobilités et pratiques spatiales dans les camps de réfugiés palestiniens au Liban", Asylon(s), n 5 [http://terra.rezo.net/article802.html] - Doraï M. K., 2008. "Itineraries of Palestinian refugees: Kinship as resource in emigration", in S. Hanafi, ed. Crossing borders, shifting boundaries: Palestinian Dilemmas. Cairo Papers in Social Science, American University in Cairo : 85-104.

- Doraï, M. K., 2007. "Les mutations récentes de l'espace migratoire syro-libanais", Revue des mondes musulmans et de la Méditerranée, $\mathrm{n}^{\circ}$ 119-120 :139-155. 